\title{
Hyponormality on a Weighted Bergman Space
}

\author{
Houcine Sadraoui $(\mathbb{D}$, Borhen Halouani $\mathbb{D}$, Mubariz T. Garayev $\mathbb{D}$, and Adel AlShehri
}

Department of Mathematics, College of Science, King Saud University, P.O. Box 2455, Riyadh 11451, Saudi Arabia

Correspondence should be addressed to Borhen Halouani; halouani@ksu.edu.sa

Received 4 May 2020; Accepted 14 July 2020; Published 5 August 2020

Academic Editor: Sivaram K. Narayan

Copyright (c) 2020 Houcine Sadraoui et al. This is an open access article distributed under the Creative Commons Attribution License, which permits unrestricted use, distribution, and reproduction in any medium, provided the original work is properly cited.

\begin{abstract}
A bounded Hilbert space operator $T$ is hyponormal if $T^{*} T-T T^{*}$ is a positive operator. We consider the hyponormality of Toeplitz operators on a weighted Bergman space. We find a necessary condition for hyponormality in the case of a symbol of the form $f+\bar{g}$ where $f$ and $g$ are bounded analytic functions on the unit disk. We then find sufficient conditions when $f$ is a monomial.
\end{abstract}

\section{Introduction}

Let $D_{R}$ denote the disk of radius $R$ in the complex plane, $d$ $\mu_{\alpha}(z)=\left(1 / \pi R^{2}\right)\left(1-|z / R|^{2}\right)^{\alpha} d A(z)$ where $d A(z)$ is the Lebesgue measure on $D_{R}$ and $\alpha>-1$. $L^{2}\left(D_{R}, d \mu(z)\right)$ denotes the Hilbert space of complex-valued functions on $D_{R}$ that are square integrable with respect to $\mu$. We write $\|f\|^{2}=\int_{D_{R}}$ $|f(z)|^{2} d \mu(z)$. When $f$ is analytic on $D_{R}$, we have

$$
f(z)=\sum_{n=0}^{\infty} a_{n} z^{n}, \quad\|f\|^{2}=\sum_{n=0}^{\infty} R^{2 n} \frac{n ! \Gamma(\alpha+1)}{\Gamma(n+\alpha+2)}\left|a_{n}\right|^{2} .
$$

Denote by $A_{\alpha}^{2}$ the space of analytic functions on $D_{R}$ such that $\|f\|^{2}<\infty$. It is known that $A_{\alpha}^{2}$ is a Hilbert space [1], and an orthonormal basis is given by $e_{n}(z)=\left(\sqrt{\Gamma(n+\alpha+2)} / R^{n}\right.$ $\sqrt{n ! \Gamma(\alpha+1)}) z^{n}$. The Toeplitz operator with symbol $f$ on $A_{\alpha}^{2}$ is defined by $T_{f}(k)=P(f k)$ where $f$ is bounded and measurable on $D_{R}, k$ is in $A_{\alpha}^{2}$, and $P$ is the orthogonal projection of $L^{2}\left(D_{R}, d \mu_{\alpha}(z)\right)$ onto $A_{\alpha}^{2}$. Hankel operators are defined by $H_{f}(k)=(I-P)(f k), f$ and $k$ as before. Recall that a bounded operator $T$ on a Hilbert space is hyponormal if $T^{*} T-T T^{*}$ is a positive operator. Hyponormality on the Hardy space is studied by Cowen in $[2,3]$. Unweighted Bergman spaces on the unit disk $(\alpha=0, R=1)$ and Toeplitz operators on these spaces were considered in $[4,5]$ and [6]. Hyponormality was first considered by Sadraoui in [7]. An improvement of the necessary condition is due to Ahern and Cuckovic [8]. A new necessary condition in a special case is found by Cuckovic and Curto in [9]. Many results on hyponormality on weighted Bergman spaces treat special cases. We cite for example $[1,10]$. In this work for simplicity's sake, we consider the case $\alpha=1$ and $R=1$. Under a smoothness assumption, we give a fairly general necessary condition for the hyponormality of Toeplitz operators with a symbol of the form $f+\bar{g}$ where $f$ and $g$ are bounded and analytic. We also give sufficient conditions when $f$ is a monomial and $g$ a polynomial without assuming $R=1$.

We begin by recalling some general properties of Toeplitz operators.

\section{Some General Properties}

We assume $f, g$ are in $L^{\infty}\left(D_{R}\right)$. Then, we have:

(1) $T_{f+g}=T_{f}+T_{g}$

(2) $T_{f}^{*}=T_{\bar{f}}$

(3) $T_{\bar{f}} T_{g}=T_{\bar{f} g}$ if $f$ or $g$ analytic on $D_{R}$ 
The use of these properties leads to describing hyponormality in more than one form. These are easy to prove by using Douglas lemma [11].

Proposition 1. Let $f, g$ be bounded and analytic on $D_{R}$. Then, the following are equivalent: [label=)]

(i) $T_{f+\bar{g}}$ is hyponormal

(ii) $H_{\bar{g}}^{*} H_{\bar{g}} \leq H_{\bar{f}}^{*} H_{\bar{f}}$

(iii) $\|(I-P)(\bar{g} k)\| \leq\|(I-P)(\bar{f} k)\|$ for any $k$ in $A_{\alpha}^{2}$

(iv) $\|\bar{g} k\|^{2}-\|P(\bar{g} k)\|^{2} \leq\|\bar{f} k\|^{2}-\|P(\bar{f} k)\|^{2}$ for any $k$ in $A_{\alpha}^{2}$

(v) $H_{\bar{g}}=\mathrm{CH}_{\bar{f}}$ where $\mathrm{C}$ is of norm less than or equal to one

We also need the following lemmas.

Lemma 2. For $s$ and $t$ integers, we have

$$
P\left(\bar{z}^{t} z^{s}\right)=\left\{\begin{array}{ll}
R^{2 t} \frac{s ! \Gamma(s-t+\alpha+2)}{\Gamma(s+\alpha+2)(s-t) !} z^{s-t}, & \text { if } s \geq t \\
0, & \text { if } s<t
\end{array} .\right.
$$

Proof. We have

$$
\begin{aligned}
\left\langle P\left(\bar{z}^{t} z^{s}\right), z^{m}\right\rangle= & \left\langle z^{s}, z^{m+t}\right\rangle=\frac{1}{\pi R^{2}} \int_{0}^{2 \pi} \int_{0}^{R} r^{s+m+t+1} e^{i(s-t-m) \theta} \\
& \cdot\left(1-\left(\frac{r}{R}\right)^{2}\right)^{\alpha} d r d \theta .
\end{aligned}
$$

We see that $\left\langle P\left(\bar{z}^{t} z^{s}\right), z^{m}\right\rangle=0$ if $s<t$. For $s \geq t,\left\langle P\left(\bar{z}^{t} z^{s}\right)\right.$, $\left.z^{m}\right\rangle \neq 0$ if and only if $m=s-t$. In this case, we get

$$
\begin{aligned}
\left\langle P\left(\bar{z}^{t} z^{s}\right), z^{s-t}\right\rangle & =\frac{2}{R^{2}} \int_{0}^{R} r^{2 s+1}\left(1-\left(\frac{r}{R}\right)^{2}\right)^{\alpha} d r \\
& =2 R^{2 s} \int_{0}^{1} u^{2 s+1}\left(1-u^{2}\right)^{\alpha} d u \\
& =R^{2 s} \int_{0}^{1} x^{s}(1-x)^{\alpha} d x=R^{2 s} \beta(s+1, \alpha+1) \\
& =R^{2 s} \frac{\Gamma(s+1) \Gamma(\alpha+1)}{\Gamma(s+\alpha+2)} .
\end{aligned}
$$

On the other hand, if $P\left(\bar{z}^{t} z^{s}\right)=\lambda z^{s-t}$, then

$$
\left\langle P\left(\bar{z}^{t} z^{s}\right), z^{s-t}\right\rangle=\lambda\left\|z^{s-t}\right\|^{2}
$$

so $\lambda=\left\langle P\left(\bar{z}^{t} z^{s}\right), z^{s-t}\right\rangle /\left\|z^{s-t}\right\|^{2}$. A computation shows

$$
\left\|z^{s-t}\right\|^{2}=R^{2(s-t)} \frac{(s-t) ! \Gamma(\alpha+1)}{\Gamma(s-t+\alpha+2)},
$$

so $\lambda=R^{2 t}(s ! \Gamma(s-t+\alpha+2) /(s-t) ! \Gamma(s+\alpha+2))$, and the result follows.

Lemma 3. If $f=\sum_{n=0}^{\infty} a_{n} z^{n}$ is bounded analytic on the disk $D_{R}$, then the $(i, j)$ th entry of the matrix of $H_{\bar{f}}^{*} H_{\bar{f}}$ with respect to the orthonormal basis $\left\{\left(\sqrt{\Gamma(n+\alpha+2)} / R^{n} \sqrt{n ! \Gamma(\alpha+1)}\right) z^{n}\right\}_{n \geq 0}$ is given by:

$$
\begin{aligned}
& \frac{\sqrt{\Gamma(j+\alpha+2)}}{R^{j} \sqrt{j !}} \frac{R^{i} \sqrt{\Gamma(i+\alpha+2)}}{\sqrt{i !}} \sum_{m \geq j-i, m \geq 0} a_{m+i-j} \overline{a_{m}} R^{2 m} \\
& \cdot \frac{(m+i) !}{\Gamma(m+i+\alpha+2)}-\frac{\sqrt{j !} \sqrt{i !} R^{j-i}}{\sqrt{\Gamma(j+\alpha+2)} \sqrt{\Gamma(i+\alpha+2)}} \\
& \cdot \sum_{0 \leq m, i-j \leq m \leq i} R^{2 m} a_{m} \overline{a_{m+j i}} \frac{\Gamma(i-m+\alpha+2)}{(i-m) !} .
\end{aligned}
$$

Proof. We have:

$$
\begin{aligned}
\bar{f} f & \frac{\sqrt{\Gamma(j+\alpha+2)}}{R^{j} \sqrt{j ! \Gamma(\alpha+1)}} z^{j} \\
& =\frac{\sqrt{\Gamma(j+\alpha+2)}}{R^{j} \sqrt{j ! \Gamma(\alpha+1)}} \sum_{n, m}^{\infty} a_{n} \overline{a_{m}} z^{n+j} \bar{z}^{m} \\
& =\frac{\sqrt{\Gamma(j+\alpha+2)}}{R^{j} \sqrt{j ! \Gamma(\alpha+1)}} \sum_{p \geq j, m \geq 0} a_{p-j} \overline{a_{m}} \bar{z}^{m} z^{p} .
\end{aligned}
$$

Thus, by Lemma 2 ,

$$
\begin{aligned}
T_{\bar{f}} T_{f} & \left(\frac{\sqrt{\Gamma(j+\alpha+2)}}{R^{j} \sqrt{j ! \Gamma(\alpha+1)}} z^{j}\right) \\
= & \frac{\sqrt{\Gamma(j+\alpha+2)}}{R^{j} \sqrt{j ! \Gamma(\alpha+1)}} \sum_{p \geq j, p \geq m, m \geq 0} a_{p-j} \overline{a_{m}} R^{2 m} \\
& \cdot \frac{p ! \Gamma(p-m+\alpha+2)}{\Gamma(p+\alpha+2)(p-m) !} z^{p-m} \\
= & \frac{\sqrt{\Gamma(j+\alpha+2)}}{R^{j} \sqrt{j ! \Gamma(\alpha+1)}} \sum_{\mathrm{p} \geq j, m \geq 0, p \geq m} a_{p-j} \overline{a_{m}} R^{2 m} \\
& \cdot \frac{p ! \Gamma(p-m+\alpha+2)}{\Gamma(p+\alpha+2)(p-m) !} z^{p-m} .
\end{aligned}
$$


Thus, noting that $\left\langle z^{p-m}, z^{i}\right\rangle \neq 0$ if $p=m+i$, we get

$$
\begin{aligned}
\left\langle T_{\bar{f}}\right. & \left.T_{f}\left(\frac{\sqrt{\Gamma(j+\alpha+2)}}{R^{j} \sqrt{j ! \Gamma(\alpha+1)}} z^{j}\right), \frac{\sqrt{\Gamma(i+\alpha+2)}}{R^{i} \sqrt{i ! \Gamma(\alpha+1)}} z^{i}\right\rangle \\
= & \frac{\sqrt{\Gamma(j+\alpha+2)}}{R^{j} \sqrt{j ! \Gamma(\alpha+1)}} \frac{\sqrt{\Gamma(i+\alpha+2)}}{R^{i} \sqrt{i ! \Gamma(\alpha+1)}} \sum_{m \geq j-i, m \geq 0} a_{m+i-j} \overline{a_{m}} R^{2 m} \\
& \cdot \frac{(m+i) ! \Gamma(i+\alpha+2)}{\Gamma(m+i+\alpha+2) i !} R^{2 i} \frac{i ! \Gamma(\alpha+1)}{\Gamma(i+\alpha+2)} \\
= & \frac{\sqrt{\Gamma(j+\alpha+2)}}{R^{j} \sqrt{j !}} \frac{R^{\Gamma(i+\alpha+2)}}{\sqrt{i !}} \sum_{m \geq j-i, m \geq 0} a_{m+i-j} \overline{a_{m}} R^{2 m} \\
& \cdot \frac{(m+i) !}{\Gamma(m+i+\alpha+2)} .
\end{aligned}
$$

We also have by Lemma 2

$$
\begin{gathered}
P\left(\bar{f} \frac{\sqrt{\Gamma(j+\alpha+2)}}{R^{j} \sqrt{j ! \Gamma(\alpha+1)}} z^{j}\right)=\frac{\sqrt{\Gamma(j+\alpha+2)}}{R^{j} \sqrt{j ! \Gamma(\alpha+1)}} P\left(\sum_{0}^{\infty} \overline{a_{n}} \bar{z}^{n} z^{j}\right) \\
=\frac{\sqrt{\Gamma(j+\alpha+2)}}{R^{j} \sqrt{j ! \Gamma(\alpha+1)}} \sum_{0}^{j} \overline{a_{n}} R^{2 n} \frac{j ! \Gamma(j-n+\alpha+2)}{\Gamma(j+\alpha+2) \cdot(j-n) !} z^{j-n} .
\end{gathered}
$$

Then,

$$
\begin{aligned}
& \left\langle T_{\bar{f}}\left(\frac{\sqrt{\Gamma(j+\alpha+2)}}{R^{j} \sqrt{j ! \Gamma(\alpha+1)}} z^{j}\right), T_{\bar{f}}\left(\frac{\sqrt{\Gamma(i+\alpha+2)}}{R^{i} \sqrt{i ! \Gamma(\alpha+1)}} z^{i}\right)\right\rangle \\
& =\frac{\sqrt{\Gamma(j+\alpha+2)} \sqrt{\Gamma(i+\alpha+2)}}{R^{i} R^{j} \sqrt{j ! \Gamma(\alpha+2)} \sqrt{i ! \Gamma(\alpha+1)}} \sum_{m=1}^{\infty} a_{m} \overline{a_{n}}\left\langle P\left(\bar{z}^{n} z^{j}\right), P\left(\bar{z}^{m} z^{i}\right)\right\rangle .
\end{aligned}
$$

The above inner product is nonzero if $i-m=j-n \geq 0$. We get by Lemma 2

$$
\begin{aligned}
\left\langle T_{f} T_{\bar{f}}\left(\frac{\sqrt{\Gamma(j+\alpha+2)}}{R^{j} \sqrt{j ! \Gamma(\alpha+1)}} z^{j}\right), \frac{\sqrt{\Gamma(i+\alpha+2)}}{R^{i} \sqrt{i ! \Gamma(\alpha+1)}} z^{i}\right\rangle \\
=\frac{\sqrt{j !} \sqrt{i ! R^{j-i}}}{\sqrt{\Gamma(j+\alpha+2)} \sqrt{\Gamma(i+\alpha+2)}} \\
\quad \sum_{0 \leq m, i-j \leq m \leq i} R^{2 m} a_{m} \overline{a_{m+j i}} \frac{\Gamma(i-m+\alpha+2)}{(i-m) !}
\end{aligned}
$$

When $R=1$, we set $D_{1}=D$. We obtain in this case the following corollary.

Corollary 4. If $f=\sum_{n=0}^{+\infty} a_{n} z^{n}$ is bounded and analytic on the unit disk $D$, and if $\alpha=1$. Then, the matrix of $H_{\bar{f}}^{*} H_{\bar{f}}$ with respect to the orthonormal basis $\left\{\sqrt{(n+2)(n+1)} z^{n}\right\}_{n \geq 0}$ is given by

$$
\begin{aligned}
\Delta_{i, j}= & \sqrt{(j+2)(j+1)} \sqrt{(i+2)(i+1)} \\
& \cdot \sum_{m \geq j-i, m \geq 0} a_{m+i-j} \overline{a_{m}} \frac{1}{(m+i+2)(m+i+1)} \\
& -\frac{1}{\sqrt{(j+2)(j+1)} \sqrt{(i+2)(i+1)}} \\
& \cdot \sum_{0 \leq m, i-j \leq m \leq i} a_{m} \overline{a_{m+j i}}(i-m+2)(i-m+1) .
\end{aligned}
$$

Recall that the Hardy space $H^{2}$ is defined by

$$
\begin{aligned}
H^{2}=\{f & =\sum_{n=0}^{+\infty} a_{n} z^{n} \text { analytic on the } \\
& \text { unit disk such that } \left.\sum_{n=0}^{+\infty}\left|a_{n}\right|^{2}<+\infty\right\} .
\end{aligned}
$$

In the following theorem, the Toeplitz operator $T_{\left|f^{\prime}\right|^{2}}$ on $H^{2}$ is not necessarily bounded. However, its matrix $\left(\Gamma_{i, j}\right)_{i, j}$ in the usual orthonormal basis of $H^{2}$ is defined.

The following theorem is an extension of Lemma 2.4.2 [7] to the weighted Bergman space.

Theorem 5. Assume $f$ bounded analytic on the unit disk $D$ and $f^{\prime} \in H^{2}$. Let $\left(\Gamma_{i, j}\right)$ be the matrix of the Toeplitz operator on $H^{2}, T_{\left|f^{\prime}\right|^{2}}$. Then, $n^{2} \Delta_{i+n, j+n} \longrightarrow 2 \Gamma_{i, j}$ as $n \longrightarrow \infty$.

Proof. The symmetric matrix of $H_{f}^{*} H_{\bar{f}}$ with respect to the orthonormal basis $\left\{\sqrt{(n+2)(n+1)} z^{n}\right\}_{n \geq 0}$ is given by (set $j$ $=i+p$ in Corollary 4 with $p \geq 0$.)

$$
\begin{aligned}
\Delta_{i, i+p}= & \sqrt{(i+p+2)(i+p+1)} \sqrt{(i+2)(i+1)} \\
& \cdot \sum_{m \geq p} a_{m-p} \overline{a_{m}} \frac{1}{(m+i+2)(m+i+1)} \\
& -\frac{1}{\sqrt{(i+p+2)(i+p+1)} \sqrt{(i+2)(i+1)}} \\
& \cdot \sum_{0 \leq m \leq i} a_{m} \overline{a_{m+p}}(i-m+2)(i-m+1) .
\end{aligned}
$$

To simplify, set $C_{n, i, p}=(i+n+1)(i+n+2)(i+n+p+1)$ $(i+n+p+2)$. Then,

$$
\begin{aligned}
\Delta_{i+n, i+n+p}= & \sqrt{C_{n, i, p}} \sum_{m \geq p} a_{m-p} \overline{a_{m}} \frac{1}{(m+i+n+2)(m+i+n+1)} \\
& -\frac{1}{\sqrt{C_{n, i, p}}} \sum_{0 \leq m \leq i+n} \mathrm{a}_{m} \overline{a_{m+p}}(i+n-m+2) \\
& \cdot(i+n-m+1)
\end{aligned}
$$


for any integer $n$. Set $m=p+l$ in the first sum, and $m=l$ in the second. With some obvious notations, we get

$$
\begin{aligned}
\Delta_{i+n, i+n+p}= & \sqrt{C_{n, i, p}} \sum_{l \geq 0} a_{l} \overline{a_{p+l}} \frac{1}{(p+l+i+n+2)(p+l+i+n+1)}-\frac{1}{\sqrt{C_{n, i, p}}} \sum_{0 \leq l \leq i+n} a_{l} \overline{a_{p+l}}(i+n-l+2)(i+n-l+1) \\
= & \sum_{l \leq i+n} a_{l} \overline{a_{p+l}} \frac{C_{n, i, p}-(i+n-l+2)(i+n-l+1)(p+l+i+n+2)(p+l+i+n+1)}{\sqrt{C_{n, i, p}}(p+l+i+n+2)(p+l+i+n+1)} \\
& +\sqrt{C_{n, i, p}} \sum_{l \geq i+n+1} a_{l} \overline{a_{p+l}} \frac{1}{(p+l+i+n+2)(p+l+i+n+1)}=\sum_{l \leq i+n} A_{n, l, p, i} a_{l} \overline{a_{p+l}}+\sum_{l \geq i+n+1} B_{n, l, p, i} a_{l} \overline{a_{p+l}} .
\end{aligned}
$$

A computation shows that

$$
A_{n, l, p, i}=\frac{l(l+p)\left((i+n+1)^{2}+(i+n+2)^{2}\right)+l p(l+p)(2 n+2 i+3)+l^{2}(l+p)^{2}}{\sqrt{C_{n, i, p}}(p+l+i+n+2)(p+l+i+n+1)} .
$$

It is not difficult to see that

$$
n^{2} A_{n, l, p, i} a_{l} \overline{a_{p+l}} \underset{n \rightarrow \infty}{\longrightarrow} 2 l(l+p) a_{l} \overline{a_{p+l}},
$$

and that the following inequality holds:

$$
\begin{aligned}
n^{2} A_{n, l, p, i}\left|a_{l} \overline{a_{p+l}}\right| & \leq 5 l(l+p)\left|a_{l}\right|\left|a_{p+l}\right| \\
& \leq 5 / 2\left(l^{2}\left|a_{l}\right|^{2}+(l+p)^{2}\left|a_{l+p}\right|^{2}\right)
\end{aligned}
$$

for $l \leq i+n$. We have that $\sum l^{2}\left|a_{l}\right|^{2}+(l+p)^{2}\left|a_{l+p}\right|^{2}<\infty$ since $f^{\prime} \in H^{2}$. Writing the sum $\sum_{l \leq i+n} n^{2} A_{n, l, p, i} a_{l} \overline{a_{p+l}}$ as an integral, where the measure is the counting measure, and using the dominated convergence theorem, we get that

$$
\sum_{l \leq i+n} n^{2} A_{n, l, p, i} a_{l} \overline{a_{p+l}} \underset{n \rightarrow \infty}{\longrightarrow} 2 \sum l(l+p) a_{l} \overline{a_{l+p}} .
$$

Clearly, $\sqrt{C_{n, i, p}} \leq(i+n+p+1)(i+n+p+2)$. So we have for $l \geq i+n+1$,

$$
\begin{aligned}
n^{2}\left|B_{n, l, p, i} a_{l} \overline{a_{l+p}}\right| & =\frac{n^{2} \sqrt{C_{n, i, p}}}{(p+l+i+n+2)(p+l+i+n+1)}\left|a_{l} \overline{a_{l+p}}\right| \\
& \leq l(l+p)\left|a_{l}\right|\left|a_{l+p}\right|
\end{aligned}
$$

for $l \geq i+n+1$. Thus,

$$
\begin{aligned}
& \sum_{l \geq i+n+1} n^{2}\left|B_{n, l, p, i}\right|\left|a_{l}\right|\left|a_{l+p}\right| \\
& \quad \leq \sum_{l \geq i+n+1} l(l+p)\left|a_{l}\right|\left|a_{l+p}\right| \\
& \quad \leq \frac{1}{2} \sum_{l \geq i+n+1} l^{2}\left|a_{l}\right|^{2}+\frac{1}{2} \sum_{l \geq i+n+1}(l+p)^{2}\left|a_{l+p}\right|^{2} .
\end{aligned}
$$

This shows that

$$
\sum_{l \geq i+n+1} n^{2} B_{n, l, p, i} a_{l} \overline{a_{p+l}} \underset{n \rightarrow \infty}{\longrightarrow} 0 .
$$
$2 \Gamma_{i, i+p}$.

\section{The Results}

Using the previous theorem leads us to our main result which extends Theorem 5.4 .3 [7].

Theorem 6. Let $f$ and $g$ be bounded and analytic on the unit disk $D$, and such that $f^{\prime} \in H^{2}$. If $T_{f+\bar{g}}$ is hyponormal on $A_{1}^{2}$ then $g^{\prime} \in H^{2}$, and $\left|g^{\prime}\right| \leq\left|f^{\prime}\right|$ a.e. on the unit circle.

Proof. Set $g=\sum_{n} b_{n} z^{n}$. All diagonal terms of the matrix of $H_{\bar{f}}^{*} H_{\bar{f}}-H_{\bar{g}}^{*} H_{\bar{g}}$ are positive; thus, using the same notation as in the previous proof, we have 


$$
\begin{aligned}
& \sum_{l \leq i+n} n^{2} A_{n, l, 0, i}\left|a_{l}\right|^{2}+\sum_{l \geq i+n+1} n^{2} B_{n, l, 0, i}\left|a_{l}\right|^{2} \\
& \quad \geq \sum_{l \leq i+n} n^{2} A_{n, l, 0, i}\left|b_{l}\right|^{2}+\sum_{l \geq i+n+1} n^{2} B_{n, l, 0, i}\left|b_{l}\right|^{2} .
\end{aligned}
$$

We have seen that the left hand side tends to $2 \sum l^{2}\left|a_{l}\right|^{2}$, a finite sum since $f^{\prime} \in H^{2}$. Writing $\sum_{l<i+n} n^{2} A_{n, l, 0, i}\left|b_{l}\right|^{2}$ as an integral with respect to the counting measure, noticing that $\chi_{\{0, . . i+n\}}(l) n^{2} A_{n, l, 0, i}\left|b_{l}\right|^{2} \underset{n \rightarrow \infty}{\longrightarrow} 2 l^{2}\left|b_{l}\right|^{2}$, and using Fatou's lemma, we deduce that $2 \sum l^{2}\left|b_{l}\right|^{2} \leq 2 \sum l^{2}\left|a_{l}\right|^{2}$ and $g^{\prime} \in H^{2}$. If $\left(\Omega_{i, j}\right)$ denotes the matrix of $H_{\bar{f}}^{*} H_{\bar{f}}-H_{\bar{g}}^{*} H_{\bar{g}}$, then $n^{2} \Omega_{i+n, j+n}$ $\longrightarrow \Lambda_{i, j}$, where $\left(\Lambda_{i, j}\right)$ is the matrix of the Toeplitz operator

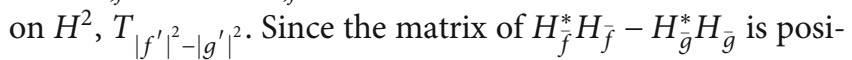
tive by hypothesis, it follows that the matrix of $T_{\left|f^{\prime}\right|^{2}-\left|g^{\prime}\right|^{2}}$ is also positive. A Toeplitz matrix is positive if its symbol is positive [12]. It follows that $\left|f^{\prime}\right| \geq\left|g^{\prime}\right|$ a.e on the unit circle.

To obtain sufficient conditions when $f$ is a monomial, we need the following lemma.

Lemma 7. Let $q$ be a positive integer, the matrix of $H_{\bar{z}^{q}}^{*} H_{\bar{z}^{q}}$ is diagonal and given by:

$d_{i}=\left\{\begin{array}{ll}R^{2 q} \frac{(q+i) ! \Gamma(i+\alpha+2)}{i ! \Gamma(q+i+\alpha+2)}, & \text { if } i<q \\ R^{2 q}\left(\frac{(q+i) ! \Gamma(i+\alpha+2)}{i ! \Gamma(q+i+\alpha+2)}-\frac{i ! \Gamma(i-q+\alpha+2)}{(i-q) ! \Gamma(i+\alpha+2)}\right), & \text { if } i \geq q\end{array}\right.$.

In what follows, we will take $\alpha=1$. It follows from Proposition 1 that, if $p>q$, the hyponormality of $T_{z^{q}+\lambda \overline{z^{p}}}$ is equivalent to the following inequalities:

$$
\begin{aligned}
&|\lambda|^{2} \frac{(i+2)(i+1)}{(p+i+2)(p+i+1)} \leq R^{2(q-p)} \frac{(i+2)(i+1)}{(q+i+2)(q+i+1)} \quad i<q ; \\
&|\lambda|^{2} \frac{(i+2)(i+1)}{(p+i+2)(p+i+1)} \leq R^{2(q-p)\left(\frac{(i+2)(i+1)}{(q+i+2)(q+i+1)}\right.} \\
&\left.-\frac{(i-q+2)(i-q+1)}{(i+2)(i+1)}\right) \quad q \leq i<p ;
\end{aligned}
$$

$$
\begin{aligned}
|\lambda|^{2} & \left(\frac{(i+2)(i+1)}{(p+i+2)(p+i+1)}-\frac{(i-p+2)(i-p+1)}{(i+2)(i+1)}\right) \\
\leq & R^{2(q-p)}\left(\frac{(i+2)(i+1)}{(q+i+2)(q+i+1)}\right. \\
& \left.-\frac{(i-q+2)(i-q+1)}{(i+2)(i+1)}\right) \quad p \leq i .
\end{aligned}
$$

Lemma 8. The inequality (28) holds if and only if $|\lambda| \leq R^{q-p}$ $\sqrt{(p+q+1)(p+q) / 2 q(2 q+1)}$.
Proof. Set $g(i)=(p+i+2)(p+i+1) /(q+i+2)(q+i+1)$. Then, (28) is equivalent to $|\lambda| \leq R^{q-p} \min \{\sqrt{g(i)} i<q\}$. Using logarithmic differentiation, it is easy to see that $g$ decreases with $i$, the minimum is thus assumed at $i=q-1$; hence, the result follows.

Lemma 9. The inequality (29) holds if and only if

$$
|\lambda| \leq \sqrt{2} \frac{q}{p+1} R^{q-p} \sqrt{\frac{(2 p+1)\left(2 p^{2}+2 p+1-q^{2}\right)}{p(p+q)(p+q+1)}} .
$$

Proof. A computation shows that inequality (29) is equivalent to

$$
|\lambda|^{2} \leq q^{2} R^{2(q-p)} \frac{(p+i+2)(p+i+1)\left(2 i^{2}+6 i+5-q^{2}\right)}{(q+i+1)(q+i+2)(i+1)^{2}(i+2)^{2}} q \leq i<p .
$$

Using logarithmic differentiation, we can see that the right hand side of the last inequality is a decreasing function of $i$. Thus, the minimum is assumed at $i=p-1$, and this proves the lemma.

A similar argument shows the following.

Lemma 10. The inequality (30) holds if and only if $|\lambda| \leq$ $(q / p) R^{q-p}$.

Proposition 11. If $q<p$, then $T_{z^{q}+\lambda \overline{z^{p}}}$ is hyponormal if and only if $|\lambda| \leq(q / p) R^{q-p}$.

Proof. Using elementary computations and the previous lemma, it is easy to see that if $|\lambda| \leq(q / p) R^{q-p}$, then inequalities (28) and (29) are satisfied. This proves the result.

Remark 12. The result remains true if $p=q$.

Denote by $U_{1}$ the unit ball of $A_{1}^{2 \perp}$.

Definition 13. For $f \in A_{1}^{2}$, we denote by $G_{f}$ the set

$$
G_{f}=\left\{g \in A_{1}^{2}: \sup _{l \in U_{1}}|\langle\bar{g}, \bar{k} l\rangle| \leq \sup _{l \in U_{1}}|\langle\bar{f}, \bar{k} l\rangle| \text { forany } k \in H^{\infty}\right\} .
$$

We see, from the density of $H^{\infty}$ in $A_{1}^{2}$ and Proposition 1 , that when $g$ and $f$ are in $H^{\infty}, g \in G_{f}$ is equivalent to $T_{f+\bar{g}}$ is hyponormal. The following proposition lists some properties of $G_{f}$ [7]. We provide the proof for the sake of completeness.

Proposition 14. For $f \in A_{1}^{2}$ the following holds: [label=()]

(a) $G_{f}$ is convex and balanced

(b) If $g \in G_{f}$, then $g+c$ is in $G_{f}$ for any complex number $c$

(c) $f \in G_{f}$. 
(d) $G_{f}$ is closed in the weak topology of $L^{2}\left(D_{R}, d \mu_{1}(z)\right)$

Proof. Only (d) needs to be verified. Assume $\left(g_{\mu}\right)$ is a net in $G_{f}$ and $g_{\mu} \longrightarrow g$. We have for $l_{0}$ in $U_{1},\left|\left\langle\bar{g}, \bar{k} l_{0}\right\rangle\right|=\lim \mid\left\langle\overline{g_{\mu}}, \bar{k}\right.$ $\left.l_{0}\right\rangle \mid$ and $\left|\left\langle\bar{g}_{\mu}, \bar{k} l_{0}\right\rangle\right| \leq \sup \left\{\left|\left\langle\bar{g}_{\mu}, \bar{k} l\right\rangle\right|, l \in U_{1}\right\} \leq \sup \{|\langle\bar{f}, \bar{k} l\rangle|$, $\left.l \in U_{1}\right\}$. The result follows by taking the limit and taking the supremum on $U_{1}$.

Using the two previous propositions, we obtain the following.

Theorem 15. Let $\left(\lambda_{n}\right)$ be a sequence of complex numbers satisfying $\sum_{q}^{\infty}\left|\lambda_{n}\right| \leq 1$ where $q$ is a positive integer. Then, $T_{z^{q}+\sum_{q}^{\infty} \lambda_{n}(q / n) R^{q-n} \overline{z^{n}}}$ is hyponormal.

We now consider the hyponormality of $T_{z^{q}+\lambda \overline{z^{p}}}$ when $p$ $<q$. In this case, hyponormality is equivalent to the following three inequalities:

$$
\begin{aligned}
|\lambda|^{2} & \leq R^{2(q-p)} \frac{(p+i+2)(p+i+1)}{(q+i+2)(q+i+1)} \quad i<p, \\
|\lambda|^{2} & \leq \frac{1}{p^{2}} R^{2(q-p)} \frac{(p+i+2)(p+i+1)(i+2)^{2}(i+1)^{2}}{(q+i+2)(q+i+1)\left(2 i^{2}+6 i+5-p^{2}\right)} \quad p \leq i<q, \\
|\lambda|^{2} & \leq \frac{q^{2}}{p^{2}} R^{2(q-p)} \frac{(p+i+2)(p+i+1)\left(2 i^{2}+6 i+5-q^{2}\right)}{(q+i+2)(q+i+1)\left(2 i^{2}+6 i+5-p^{2}\right)} \quad q \leq i .
\end{aligned}
$$

A computation, using logarithmic differentiation, allows us to see that the right hand side of each of these inequalities increases with $i$. This leads to the following inequalities:

$$
\begin{aligned}
& |\lambda| \leq R^{q-p} \sqrt{\frac{(p+2)(p+1)}{(q+2)(q+1)}} ; \\
& |\lambda| \leq R^{q-p} \frac{(p+1)(p+2)}{p} \sqrt{\frac{2(2 p+1)}{(p+q+1)(p+q+2)(p+5)}} ; \\
& |\lambda| \leq R^{q-p} \frac{q}{p} \sqrt{\frac{(q+5)(p+q+1)(p+q+2)}{2\left(2 q^{2}+6 q+5-p^{2}\right)(2 q+1)}}
\end{aligned}
$$

Set

$$
\begin{aligned}
& A_{p, q}=\sqrt{\frac{(p+2)(p+1)}{(q+2)(q+1)}}, \\
& B_{p, q}=\frac{(p+1)(p+2)}{p} \sqrt{\frac{2(2 p+1)}{(p+q+1)(p+q+2)(p+5)}}, \\
& C_{p, q}=\frac{q}{p} \sqrt{\frac{(q+5)(p+q+1)(p+q+2)}{2\left(2 q^{2}+6 q+5-p^{2}\right)(2 q+1)}} .
\end{aligned}
$$

Write

$$
B_{p, q}=\sqrt{\frac{(2 p+1)(2 p+2)(p+1)\left(p^{2}+4 p+4\right)}{(p+q+1)(p+q+2) p^{2}(p+5)}} .
$$

Since $g(i)=(p+i+2)(p+i+1) /(q+i+2)(q+i+1)$ increases with $i$, we get

$$
\frac{(p+1)(p+2)}{(q+1)(q+2)} \leq \frac{(2 p+1)(2 p+2)}{(p+q+1)(p+q+2)} .
$$

Also, $(p+1)\left(p^{2}+4 p+4\right) \geq p^{2}(p+5)$. Thus, $A_{p, q}<B_{p, q}$ for $p<q$. We have

$$
C_{p, q}=\sqrt{\frac{q^{2}(p+q+2)(p+q+1)(q+1)(q+5)}{2 p^{2}(q+1)(2 q+1)\left(2 q^{2}+6 q+5-p^{2}\right)}},
$$

and we can readily verify that for $p<q$, we have

$$
q^{2}(q+1)(q+5) \geq p^{2}\left(2 q^{2}+6 q+5-p^{2}\right)
$$

As before, $g(i)=(p+i+2)(p+i+1) /(q+i+2)(q+i+$ 1 ) increases with $i$ if $p<q$. We get $g(0) \leq g(q)$ which leads to the inequality

$$
\frac{(p+2)(p+1)}{(q+1)(q+2)} \leq \frac{(p+q+2)((p+q+1)}{2(q+1)(2 q+1)} .
$$

Combining the last inequality with inequality (40), we deduce the following:

Proposition 16. If $p<q$ then $T_{z^{q}+\lambda \overline{z^{p}}}$ is hyponormal if and only if $|\lambda| \leq R^{q-p} \sqrt{(p+2)(p+1) /(q+2)(q+1)}$.

Remark 17. The result holds also if $p=q$.

From the properties of $G_{z^{q}}$, noted in Proposition 14, we deduce the following corollaries.

Corollary 18. Let $\lambda_{1}, \lambda_{2} \ldots, \lambda_{q}$ be complex numbers satisfying $\sum_{1}^{q}\left|\lambda_{i}\right| \leq 1$. Then, $T_{z^{q}+\sum_{1}^{q} \lambda_{n} R^{q-n} \sqrt{(n+2)(n+1) /(q+2)(q+1)} \overline{z^{n}}}$ is hyponormal.

Corollary 19. Let $\left(\lambda_{n}\right)$ be a sequence of complex numbers satisfying $\quad \sum_{1}^{\infty}\left|\lambda_{i}\right| \leq 1 . \quad$ Then, $T_{z^{q}+\sum_{1}^{q} \lambda_{n} R^{q-n} \sqrt{(n+2)(n+1) /(q+2)(q+1)} \overline{z^{n}}+\sum_{q+1}^{\infty} \lambda_{n}(q / n) R^{q-n} z^{\bar{n}}}$ is hyponormal.

\section{Data Availability}

No data were used to support this study.

\section{Conflicts of Interest}

The authors declare that are no conicts of interest regarding the publication of this paper. 


\section{Acknowledgments}

The authors would like to extend their sincere appreciation to the Deanship of scientic research at King Saud University for its funding of this work through Research Group Project no RG-1439-68.

\section{References}

[1] M. Hazarika, "On hyponormality of Toeplitz operators on the weighted Bergman space," Communications in Mathematics and Applications, vol. 3, no. 2, pp. 147-157, 2012.

[2] C. Cowen, "Hyponormal and subnormal Toeplitz operators, in Surveys of Some Results in Operator Theory," in Pitman Research Notes in Math, Vol, 171, pp. 155-167, Longman, Harlow, 1988.

[3] C. C. Cowen, "Hyponormality of Toeplitz operators," Proceedings of the American Mathematical Society, vol. 103, no. 3, pp. 809-812, 1988.

[4] S. Axler, "Bergman spaces and their operators, in Surveys of Some Recent results in Operator Theory," in Pitman Research Notes in Math, Vol 171, pp. 1-50, Longman, Harlow, 1988.

[5] S. Axler, "multiplication operators," Duke Mathematical Journal, vol. 53, no. 2, pp. 315-332, 1986.

[6] K. Zhu, Operator Theory in Function Spaces, Dekker, New York, 2007.

[7] H. Sadraoui, Hyponormality of Toeplitz Operators and Composition Operators, Thesis, Purdue University, 1992.

[8] P. Ahern and Z. Cuckovic, "A mean value inequality with applications to Bergman space operators," Pacific Journal of Mathematics, vol. 173, no. 2, pp. 295-305, 1996.

[9] Z. Cuckovic and R. Curto, "A new necessary condition for the hyponormality of Toeplitz operators on the Bergman space," Journal of Operator Theory, vol. 79, no. 2, pp. 287-300, 2018.

[10] S. Hwang and J. Lee, "Hyponormal Toeplitz operators on the weighted Bergman spaces," Mathematical Inequalities \& Applications, vol. 15, pp. 323-330, 2012.

[11] R. G. Douglas, "On majorization, factorization, and range inclusion of operators on Hilbert space," Proceedings of the American Mathematical Society, vol. 17, no. 2, pp. 413-432, 1966.

[12] U. Grenander and G. Szegö, Toeplitz Forms and their Applications, vol. 11, no. 10, 1958, University of California Press, Berkeley and Los Angeles, 1958. 